\title{
Die Brachlandentwicklung im Obergoms 1970-1980
}

\section{Einleitung}

Die Brachlegung von Kulturland im Schweizer Berggebiet hat in den letzten Jahrzehnten einen bedeutenden Umfang angenommen. Mehrere Institute bemühen sich heute um die Erfassung von Verbreitung, Ursachen und sowohl ökologischen als auch ökonomischen Auswirkungen. Umfassendere Arbeiten, die sich systematisch mit diesem räumlichzeitlichen Phänomen auseinandersetzen und vor allem die Dynamik dieses Landschaftsprozesses aufzeigen, fehlen jedoch weitgehend. Aus diesem Grunde veranstaltete das Geographische Institut der Universität Zürich im September 1980 eine Arbeitswoche im Goms. Ziel war es, die von GREsCH 1970 aufgenommene und in der $\mathrm{GH}$ veröffentlichte Brachlandkartierung (GRESCH 1972) mit denselben Methoden zehn Jahre später zu wiederholen. Damit wurde es möglich,

- die Veränderungen in den brachliegenden Flächen 1970-1980 als formalen Ausdruck aktualgeographischer Prozesse $\mathrm{zu}$ erfassen und $\mathrm{zu}$ erklären $\mathrm{zu}$ versuchen,

- die ablaufenden Prozesse mit den Zielvorstellungen des Regionalplanungskonzepts zu vergleichen,

- prognostische Aussagen und planerische Maßnahmen abzuleiten und

- das von Gresch (1971) entwickelte Modell auf seine Richtigkeit zu überprüfen.

Ende August 1980 konnte durch den LAD Dübendorf ein gezielter Bildflug durchgeführt werden. Diese Luftaufnahmen dienten dann als eigentliche Kartierungsgrundlage.

Am Arbeitslager beteiligten sich folgende Geographiestudenten:

Reta Caspar, Werner Hirzel, dipl. Geogr. Stefan Julen, August Keller, Gian-Carlo Keller, Walter Mahler, Regine Potocki, Markus Ruf, GianReto Schmid, URs Schori, Ueli Schwotzer.

Sie alle haben zu den nachfolgenden Ergebnissen entscheidend beigetragen und die vorliegende Arbeit sowohl durch ihren großen persönlichen Einsatz als auch durch ihre kritische Auseinandersetzung mit dem Thema überhaupt erst möglich gemacht. Dafür danken wir ihnen bestens. Unser Dank gilt aber auch der Gemeinde Niederwald, die uns ihr Schulhaus samt Küche zur Verfügung stellte, und ihrem rührigen Präsidenten, Herrn H. Mutter, der uns während der ganzen Arbeitswoche aktiv unterstützte. Ebenso danken wir der EMPA Dübendorf für die Flächenmessungen am Quantimet.

\section{Arbeitsgebiet und Kartierungsmethode}

Das 1980 bearbeitete Gebiet entspricht demjenigen der Karte von Gresch (1972). Es umfaßt also das gesamte obere Goms, von Oberwald bis Niederwald, wobei nur die Kulturflächen in der Talsohle und am Hangfuß, in der Regel also unterhalb des Waldes gelegen, einbezogen sind. Nicht berücksichtigt wurden die Alpweiden und allfällige Mähder über der Waldgrenze.

Nach Hottes (1975) untersucht die Geographie raumwirksame Prozesse und ihre Erscheinungsformen. Das Brachland soll also als Raummuster von zugrundeliegenden Prozessen verstanden und seine Entwicklung in diesem Sinne erklärt werden. Die beobachteten Prozesse sollen mit den Zielvorstellungen, die im Regionalen Entwicklungskonzept 1974 formuliert wurden, verglichen werden. Sie sind von bestimmbaren Rahmenbedingungen abhängig, so daß eine exakte Prognose der Landschaftsentwicklung sowie die Formulierung eines Maßnahmenkataloges möglich werden.

Als Kartierungsunterlage dienten die im August 1980 durch den LAD Dübendorf geflogenen Luftbilder. Ihr mittlerer Maßstab beträgt in der Talsohle ca. $1: 15000$. Sie wurden für bequemeres Arbeiten auf rund $1: 10000$ vergrößert. Durch direktes Kartieren im Feld und vom Gegenhang aus wurden die Grenzen zwischen bewirtschaftetem und nicht mehr bewirtschaftetem Areal visuell ins Luftbild eingetragen. Mit Hilfe des Taschenstereoskops konnten die Geländeformen stereoskopisch betrachtet werden. Nachkontrollen erfolgten unmittelbar anschließend an die Feldbegehung im Stereoskop, da uns ein Arbeitsraum im alten Schulhaus Niederwald zur Verfügung stand. Ebenso lagen die Luftbilder mit der 1970er Kartierung

Pierre Walther, Harold Haefner, Geogr. Institut der Universität Zürich, Postfach, 8033 Zürich

Peter Gresch, ORL-Institut ETHZ, 8093 Zürich 
Tabelle 1 Brachlandentwicklung 1970-1980 im Obergoms

\begin{tabular}{lllllll}
\hline & $\begin{array}{l}\text { Brachland } \\
1970\end{array}$ & $\begin{array}{l}\text { Brachland } \\
1980\end{array}$ & $\begin{array}{l}\text { Brachland } \\
1970 \text { (in \% } \\
\text { des Perime.) }\end{array}$ & $\begin{array}{l}\text { Brachland } \\
1980 \text { (in } \% \\
\text { des Perime.) }\end{array}$ & $\begin{array}{l}\text { Brachland- } \\
\text { entwicklung } \\
1970-1980\end{array}$ & $\begin{array}{l}\text { Brachland- } \\
\text { entwicklung } \\
1970-1980 \\
\text { (in \% des Perime.) }\end{array}$ \\
\hline Niederwald & 23,3 & 27,4 & $28 \%$ & $33 \%$ & $+4,1$ & $+5 \%$ \\
Blitzingen & 74,0 & 41,2 & $46 \%$ & $27 \%$ & $-32,9$ & $-19 \%$ \\
Selkingen & 34,2 & 11,2 & $35 \%$ & $11 \%$ & $-23,0$ & $-24 \%$ \\
Biel & 12,3 & 7,1 & $17 \%$ & $10 \%$ & $-5,1$ & $-7 \%$ \\
Ritzingen & 11,8 & 8,0 & $13 \%$ & $9 \%$ & $-3,8$ & $-4 \%$ \\
Gluringen & 12,0 & 11,2 & $12 \%$ & $11 \%$ & $-0,8$ & $-1 \%$ \\
Reckingen & 33,2 & 23,8 & $16 \%$ & $12 \%$ & $-9,4$ & $-4 \%$ \\
Münster & 25,0 & 67,5 & $9 \%$ & $24 \%$ & $+42,5$ & $+15 \%$ \\
Geschinen & 13,6 & 14,0 & $8 \%$ & $8 \%$ & $+0,4$ & $0 \%$ \\
Ulrichen & 16,9 & 36,6 & $7 \%$ & $15 \%$ & $+19,7$ & $+8 \%$ \\
Obergesteln & 12,6 & 30,7 & $5 \%$ & $12 \%$ & $+18,1$ & $+7 \%$ \\
Oberwald & 15,0 & 39,2 & $8 \%$ & $20 \%$ & $+24,2$ & $+12 \%$ \\
\hline Obergoms total & 284,0 & 317,9 & $15 \%$ & $17 \%$ & $+33,9$ & $+2 \%$ \\
\hline
\end{tabular}

Alle Flächenangaben in ha, Prozentangaben in \% des kartierten Perimeters (GRESCH 1972)

für direkte Vergleichszwecke vor. Für brachgefallenes Areal wurden folgende Kriterien festgesetzt:

- Ackerland: Flächen sind mit hohen Stauden bedeckt.

- Wiesland: Nicht weggeführte Biomasse bildet einen Streufilz, der im Herbst am abgestorbenen Pflanzenmaterial gut sichtbar ist.

Dies ermöglichte es, nur vorübergehend nicht mehr genutztes Land auszuschließen. Die noch beweideten Flächen konnten mit Hilfe der Umzäunungen erfaßt werden.

Nachher wurden die Grenzen mit Hilfe des ZEISSLuftbildumzeichners aus dem Luftbild in eine Karte 1:10000 übertragen. Die Flächenberechnung (Tab. 1) erfolgte mittels eines Quantimets.

\section{Interpretation der Ergebnisse}

\subsection{Grundsätzliche Aspekte}

Bei der Interpretation der beiden Brachlandkarten stellen sich einige methodische Schwierigkeiten: Die Kartierung eines Phänomens ist immer zeitgebunden, und somit müssen die saisonalen und annuellen Schwankungen mitberücksichtigt werden. Im Zusammenhang mit dem Brachland sind zwei Genauigkeitsbereiche zu unterscheiden:

a) Auf ehemaligem Ackerland sind die Schwankungen relativ gering, da, wie Monheim (1956) zeigt, von jeher nach einem geregelten Fruchtwechsel bewirtschaftet wird. Wie Gerber/GianQuet (1980) in einer detaillierten Kartierung im Binntal belegen, können aber auch im Ackerland die temporalen Veränderungen beträchtlich sein. b) Größer sind die jährlichen Schwankungen bei der Bewirtschaftung des Grünlandes, insbesondere der Magerwiesen. Wichtige Faktoren bei der Brachlegung sind etwa das Wetter während der Erntezeit, die Verfügbarkeit von Helfern während der kurzen Arbeitsspitzen usw. Da es sich 1980 um ein ausgesprochenes Schlechtwetterjahr handelte, wurden vermutlich einige Parzellen weniger gemäht.

Vor allem beim quantitativen Vergleich der beiden Kartierungen (Tab. 1) müssen diese Genauigkeitsbereiche mitberücksichtigt werden. Die gemessenen Werte zeigen nur die Größenordnung der Brachlandausdehnung an; sie können aber nicht statistisch ausgewertet werden. Das Ausmaß dieser Bewirtschaftungsschwankungen ist bisher noch nirgends gemessen worden.

Ein weiteres Problem stellt sich beim Vergleich der Brachlandflächen mit den von der ESTA erhobenen Angaben. Die Realteilung hat im Obergoms zu einer extremen Aufsplitterung der einzelnen Betriebsflächen geführt. KLOPSCH (1969) zeigt, daß der Grundbesitz eines Bauern über mehrere Gemeinden verteilt sein kann. Da die Daten der ESTA auf der Basis der einzelnen Betriebe erhoben werden, können sie nicht mit den gemeindeweisen Flächendaten der Brachlandkartierung in Beziehung gesetzt werden.

Aus den beiden Brachlandkarten 1970/1980 lassen sich folgende räumliche Prozesse formulieren:

a) Zunahme des Brachlandes auf den ehemaligen Ackerbauflächen (Fig. 1).

b) Abnahme des Brachlandes im unteren Talabschnitt (Fig. 2).

Die zugehörigen raumwirksamen Prozesse sollen in den anschließenden Abschnitten dargestellt werden. 


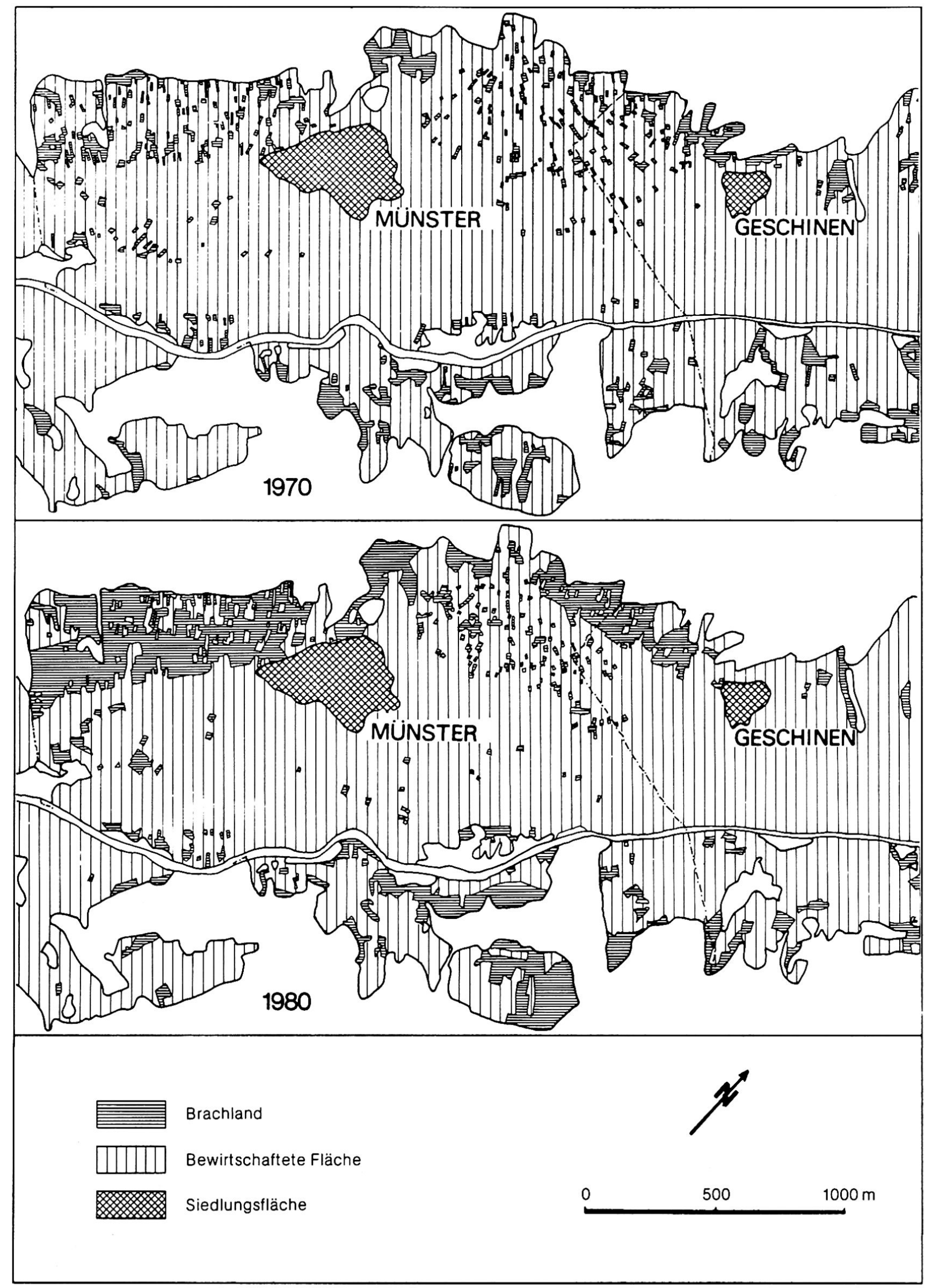

Fig. 1 Zunahme des Brachlandes in der reich parzellierten Ackerflur (Gemeinden Münster, Geschinen) 
3.2 Erster räumlicher Prozeß: Zunahme des Brachlandes auf den ehemaligen Ackerbauflächen

Im Zeitraum 1970-1980 hat das Brachland vor allem an der sonnenexponierten, reich parzellierten Talflanke und auf den Schuttfächern im ehemaligen Ackerbaugebiet zugenommen. Wie Tab. 1 zeigt, betrifft diese Entwicklung hauptsächlich den obersten Talabschnitt (Münster bis Oberwald). Fig. 1 illustriert das räumliche Verbreitungsmuster dieser Brachlandentwicklung mit einem Kartenvergleich.

Bei der Suche nach einer Erklärung für diese Veränderung des Landschaftsbildes stoßen wir auf verschiedene raumwirksame Prozesse, die nach KLOPSCH (1969) zwischen 1950 und 1960 in der Landwirtschaft einsetzten und seit 1970 weiter fortgeschritten sind. In mehreren Arbeiten zum alpinen Kulturlandschaftswandel werden diese sozio-ökonomischen Umstrukturierungen etwa als Umbruch oder Systemöffnung bezeichnet.

Grundsätzlich ist es möglich, die Entwicklung vor dem Hintergrund zweier Ansätze zu diskutieren: Die klassische Theorie vertritt die Auffassung, da $\beta$ im Berggebiet vor allem Grenzertragsböden brachfallen, weil ihre Bewirtschaftung wegen steigender Produktionskosten unrentabel wird (SURBer 1973). Bereits 1956 versuchte HARTKE aber zu zeigen, «daß das ganze Phänomen (Brachland) ein physiognomischlandwirtschaftliches Ergebnis eines sozialen Umschichtungsprozesses ist, der nach dem Kriege... in Gang gekommen ist) (HARTKE 1956, S. 259). Der erste Ansatz orientiert sich stärker an objektiv meßbaren Produktionsfaktoren der Naturlandschaft, während die sozialgeographische Theorie die Entscheidungsmechanismen der einzelnen Betriebsleiter in seine Erklärungsversuche einbezieht. Die beiden Theorien widersprechen sich aber nicht, da sie auf verschiedene Fragestellungen ausgerichtet sind und die Brachlegung von Kulturland nie von Einzelursachen, sondern von Ursachenkomplexen bestimmt wird.

Nach der klassischen Auffassung ist die Brachlegung vor allem durch die marginale Lage eines Standortes bedingt. Grenzertragslagen, seien sie nun absolut oder relativ (Moos 1979), fallen demzufolge als erste brach, wenn sich die Produktionskosten oder die Betriebsstruktur ändern. GRESCH versuchte 1971 und in einer gesamtschweizerischen Studie 1980 solche Grenzertragslagen mit Hilfe von quantitativen Modellen zu simulieren (Kap.4). Als wichtigste Faktoren bestimmte er Hangneigung, Höhendifferenz zum Dorf (Hof) und Geländeform. Diese Faktoren haben in den letzten Jahrzehnten vor dem Hintergrund einer zunehmenden Modernisierung der Landwirtschaft eine große Bedeutung erlangt. Dieser Modernisierungsprozess steht mit der Abwanderung von Arbeitskräften und der damit verbundenen Aufsplitterung der Großfamilien in Zusammenhang. Die Zahl der Landwirtschaftsbetriebe hat sich in den letzten Jahr- zehnten in allen Gemeinden des Obergoms zunehmend vermindert, wobei von 1965 bis 1975 in den Gemeinden Gluringen, Münster, Oberwald und Selkingen eine gleichzeitige Vergrößerung der verbleibenden Betriebe festzustellen ist. Arbeitskräfte wurden durch Kapital (Investition in Maschinen) ersetzt. Ein wichtiger raumwirksamer Prozeß ist somit die Mechanisierung der Landwirtschaft, die in allen Gemeinden des Obergoms zwischen 1965 und 1975 zugenommen hat. Diese maschinelle Bewirtschaftung ist an Rahmenbedingungen wie Hangneigung, Parzellengröße und Erschließung gebunden, die gerade an den steilen Talflanken nicht gegeben sind. Im ehemaligen Ackerbaugebiet verhindert weiter das dichte Netz von Bewässerungskanälen einen Einsatz von Maschinen. Roten (1969) bemerkt, daß sich vor allem die Güterzerstückelung stark nachteilig auf die Bewirtschaftungsrendite auswirkt. Es wird bedauert, daß 1940/1942 die Talflanken und Schuttfächer (Ackerbauzone) nicht in die Güterzusammenlegungen einbezogen wurden und somit der Weg für eine maschinelle Bewirtschaftung geebnet wurde. Mit Hilfe dieser Grenzertragstheorien kann also der beobachtete räumliche Prozeß teilweise erklärt werden.

Zwei Beobachtungen sind nun anzuführen: Einerseits kann man behaupten, daß die naturräumlichen Voraussetzungen für die Landwirtschaft im Obergoms selbst an den Talflanken, verglichen mit andern Bergtälern (z. B. Mattertal bei JULEN 1979), relativ gut sind, der Anteil an Brachland aber trotzdem höher ist. Andererseits erstaunt die Bemerkung eines Bauern, daß «zumindest die älteren Landwirte bei der Entscheidung zwischen Nutzung oder Brachlegung selten an den Ertrag denken». Dies führt uns zum Ansatz von HaRTKe (1956) zurück, der die Ursachen der Brachlegung in einer sozialen Umschichtung und dem damit verbundenen Wertwandel sucht. Im Obergoms ist die Frage interessant, ob ein alters- oder erwerbsspezifischer Unterschied im Bewirtschaftungsverhalten zu beobachten ist. Antwort auf diese Frage finden wir zum Teil in den Arbeiten von MatTER (1978) und Landtwing (1979), die diese Problemkreise mit Befragungen in benachbarten Gebieten untersucht haben.

Matter (1978) zeigt, daß traditionelle Werte des Autarkiekomplexes vor allem in der älteren Generation noch stark verwurzelt sind. Dabei stellt er im Lötschental einen Bruch zwischen älterer und jüngerer Generation fest. Wichtig sind «Achtung vor dem Alter», "mit dem eigenen Schicksal zufrieden sein» und religiöse Werte (MatTer 1978). Dazu gehört auch der Ackerbau. Ein modernes Wettbewerbsdenken ist erst unter der jüngeren Bauernschicht zu finden. Der Wandel der Bewirtschaftungsstruktur wurde bedingt durch Aufsplitterung der Großfamilien und Aufkommen eines «Paritätslohndenkens» (Lichtenberger 1979). Landtwing (1979) stellt fest, daß heute viele junge Bauern nicht einmal mehr die Lage ihrer Parzellen kennen. Er benutzt diesen Wandel als einen 


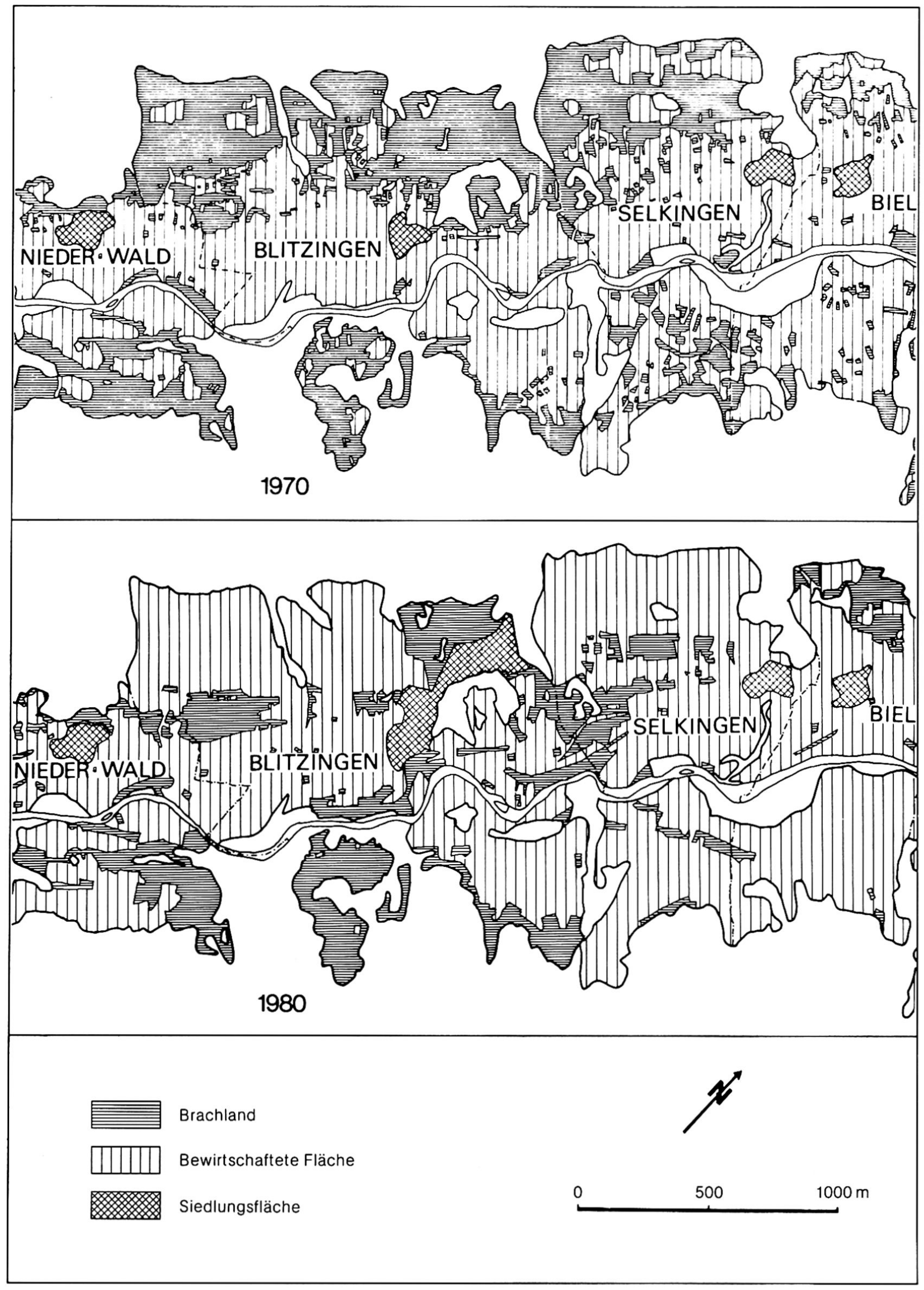

Fig. 2 Zunahme der Beweidung auf ehemaligem Brachland (Gemeinden Niederwald bis Biel) 
Indikator für die Bestimmung der «Schollenverbundenheits der Landbesitzer.

Ein weiterer Prozeß der Nachkriegszeit ist das Aufkommen der Nebenerwerbslandwirtschaft. Arbeiterbauern sind nach LANDTwING (1979) durch ein anderes Bewirtschaftungsverhalten als Vollbauern geprägt, da sie nicht mehr viel Zeit für eine arbeitsintensive Landwirtschaft zur Verfügung haben. Im Obergoms hat die Entwicklung der Nebenerwerbslandwirtschaft räumlich differenziert eingesetzt. In den untersten Gemeinden wohnen seit Jahrzehnten Arbeiterbauern, die täglich in die Industriegebiete des Mittelwallis pendeln. Die Zunahme des Brachlandes an den Talhängen ist also auch mit diesem sozialgeographischen Ansatz durch den natürlichen Rückgang der in der Tradition verhafteten Bewirtschafter erklärbar.

\subsection{Zweiter räumlicher Prozeß: Abnahme des Brachlandes im unteren Talabschnitt}

Gresch (1971) stellte einen großen Anteil an Brachland im unteren Abschnitt (Reckingen bis Niederwald) fest. Er begründete dies u. a. mit der Zunahme des Lohngefälles zwischen Industrie und Landwirtschaft, die in der Nachkriegszeit viele Bauern zur Aufgabe ihrer Betriebe verleitete. In den letzten zehn Jahren ist hier ein Rückgang des Brachlandes und ein Aufkommen extensiver Weidebewirtschaftung festzustellen (Fig. 2). Selbst steile, reich parzellierte Hänge werden heute mit Rindvieh und Schafen beweidet, wobei größere Flächen mit Elektrozäunen umgrenzt werden. Es stellt sich die Frage, weshalb dieser Prozeß in einigen Gemeinden (z. B. Reckingen) einsetzen konnte, in anderen (z. B. Münster) jedoch bis heute verhindert wird.

Die Suche nach einer Antwort führt uns zu folgenden Überlegungen: Das Einsetzen des Beweidungsprozesses ist im Obergoms von verschiedenen Rahmenbedingungen abhängig. Dabei kann man Grundvoraussetzungen, die vor allem im strukturellen Bereich liegen, und von der gewählten Beweidungsform abhängige Rahmenbedingungen unterscheiden. Eine wichtige Grundvoraussetzung ist das Vorhandensein von beweidbaren Parzellen, die eine gewisse Mindestgröße aufweisen sollten. Im reich parzellierten Wiesund Ackerland, wo die Parzellenbindung des Bewirtschafters noch gro $\beta$ ist, ist Beweidung undenkbar. Dieser Prozeß wird offenbar nur möglich, wenn die Brachlegung praktisch bis zu einem totalen Stadium fortgeschritten ist und damit die Bereitschaft zur Verpachtung entsteht. Nach der Lösung dieser Parzellenbindung beobachtete auch LANDTwING (1979), daß sich Meinungen wie «besser noch beweiden, als brachliegen lassen" in der Dorfgemeinschaft durchsetzten. Eine zweite Grundvoraussetzung ist eine gewisse Nachfrage nach Weideland, die die Bereitschaft zu Innovationen, wie sie Pachtlandarrondierungen erfordern, sei es auch nur auf der Basis mündlicher Abmachungen, verstärkt. So ist die Beweidung von ehemaligem Ackerland im Zusammenhang mit der Krise der Alpwirtschaft zu sehen. Vielerorts fehlen geeignete Kuhalpen (Reg. Entw. Konzept 1976), so $\mathrm{da} B$ die unteren Hanglagen beweidet werden. Eine Grundvoraussetzung ist aber vor allem der Wille der Dorfgemeinschaft zur Zusammenarbeit, der im Obergoms nicht eben stark ist (Reg. Entw. Konzept 1976). Die Gründe liegen wohl im Erbgesetz und der Überalterung der Bevölkerung (KLOPSCH 1969), die viele Erbstreitigkeiten vor dem Hintergrund von Baulandspekulationen (GRESCH 1971) immer wieder neu entflammen lassen.

Es können verschiedene Beweidungsformen beobachtet werden, die von unterschiedlichen Rahmenbedingungen abhängen. In Reckingen und Blitzingen wird das ehemalige Wies- und Ackerland zunehmend von Rindvieh beweidet. Einerseits bestoßen die einheimischen Bauern das Grünland mit ihrem Braunvieh, andererseits werden auch größere Flächen durch einheimische Vermittler an Bauern aus dem Mittelland verpachtet. Diese Rindviehbeweidung ist von der Hangneigung abhängig. Die geforderten Grenzwerte von $60 \%$ für Rinder (DieTl 1979) werden an manchen Hängen überschritten. Wegen der Trockenheit des Bodens ist die Hangneigung als Standortfaktor im Obergoms nicht so bedeutend wie in anderen Tälern des Schweizer Berggebietes. Wichtiger ist das Vorhandensein einer geeigneten Infrastruktur wie Stallbauten, Zufahrtswege und Tränkeanlagen. Bereits RoteN (1969) bezeichnete die Gebäudesanierung als eines der wichtigsten landwirtschaftlichen Probleme im Obergoms. Das Fehlen geeigneter Stallgebäude ist vor allem für die Beweidung der ehemaligen Ackergebiete hemmend.

In anderen Gemeinden (z.B. Selkingen) hat die Schafbeweidung im letzten Jahrzehnt stark zugenommen. Dabei wird die dorfnahe Flur in standortwechselnder Haltung, d.h. im Frühling und Herbst sowie Alpung im Sommer, beweidet. Die Zunahme dieser extensiven Schafbetriebe ist in einem engen Zusammenhang mit dem Aufschwung der Nebenerwerbslandwirtschaft zu sehen. LANDTwING (1979) zeigt, daß die Schafzucht von vielen Arbeiterbauern in der Freizeit betrieben wird. Er spricht von «Hobbybauern", bei denen das Motiv der Freizeitgestaltung wichtiger als die Erzielung eines hohen Ertrages ist. Diese Betrachtungen zeigen, weshalb die Beweidung der dorfnahen Flur im unteren Abschnitt des Obergoms einsetzte. Der Rückgang der hauptberuflichen Landwirtschaft hat hier früher eingesetzt als im oberen Talabschnitt. Die Auflösung der Parzellenbindung und das Ansteigen der Zahl der Nebenerwerbslandwirte mit ihren charakteristischen Wertvorstellungen ebneten den Weg für eine extensive Bewirtschaftung der Talhänge. 


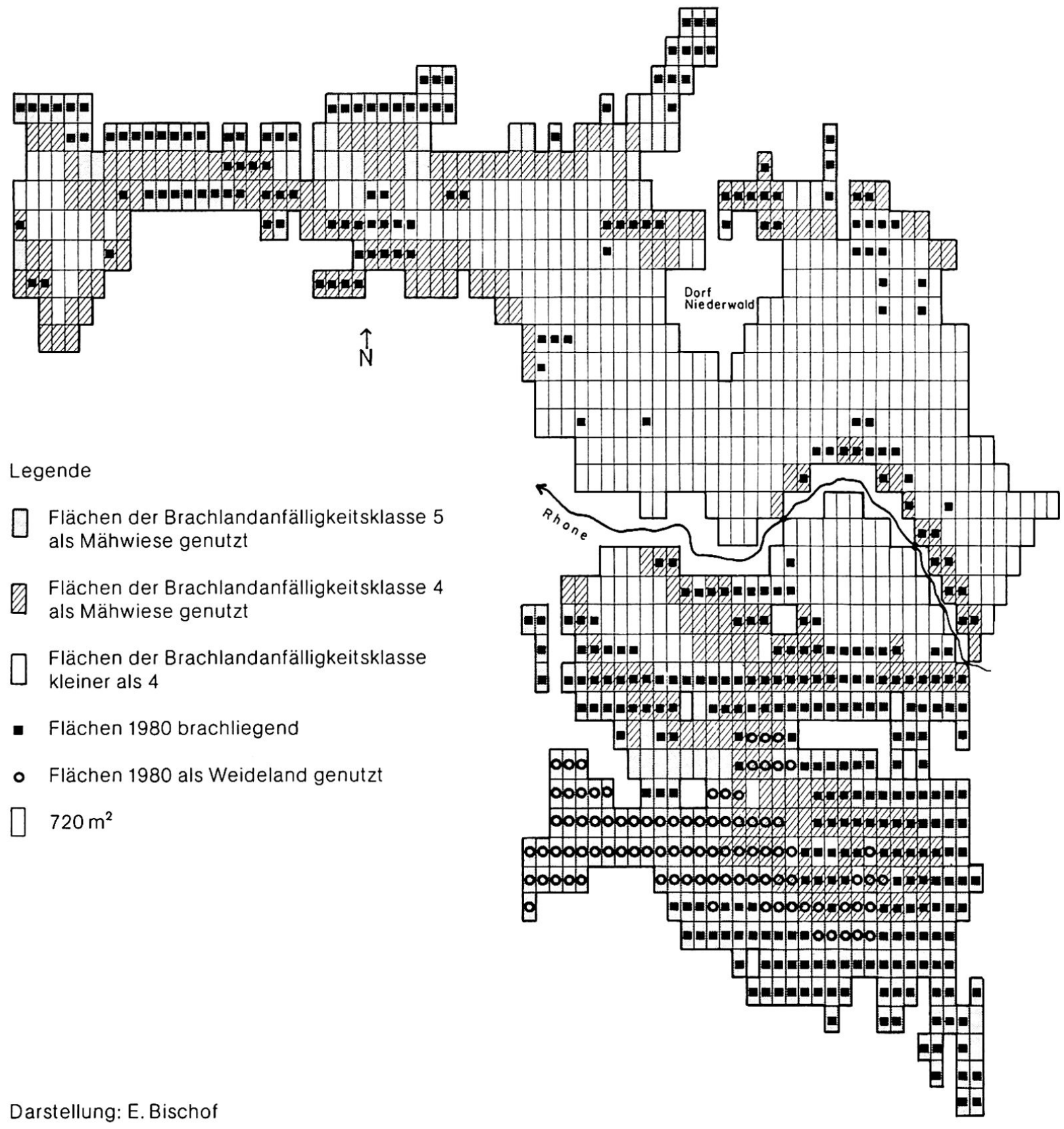

Fig. 3 Bracheanfälligkeit und Landnutzungskartierung 1980 (Gemeinde Niederwald)

\section{Erklärungsversuche mittels eines quantitativen Modells}

\subsection{Modellbeschrieb}

Die Kartierung des Brachlandes im Obergoms 1970 lie $\beta$ vermuten, daß die Brachlegung gewissen Gesetzmäßigkeiten folge. Zur Überprüfung dieser Hypothese wurde für Niederwald ein quantitatives Modell erar- beitet (GRESCH 1971). Es basiert auf folgenden thematischen Karten:

- Hangneigung

- Exposition

- Höhenlage

- Verkehrserschließung der Parzellen

- Distanz zum Dorf

- Höhendifferenz (zwischen Dorf und Parzelle)

- Abstand zum Waldrand 


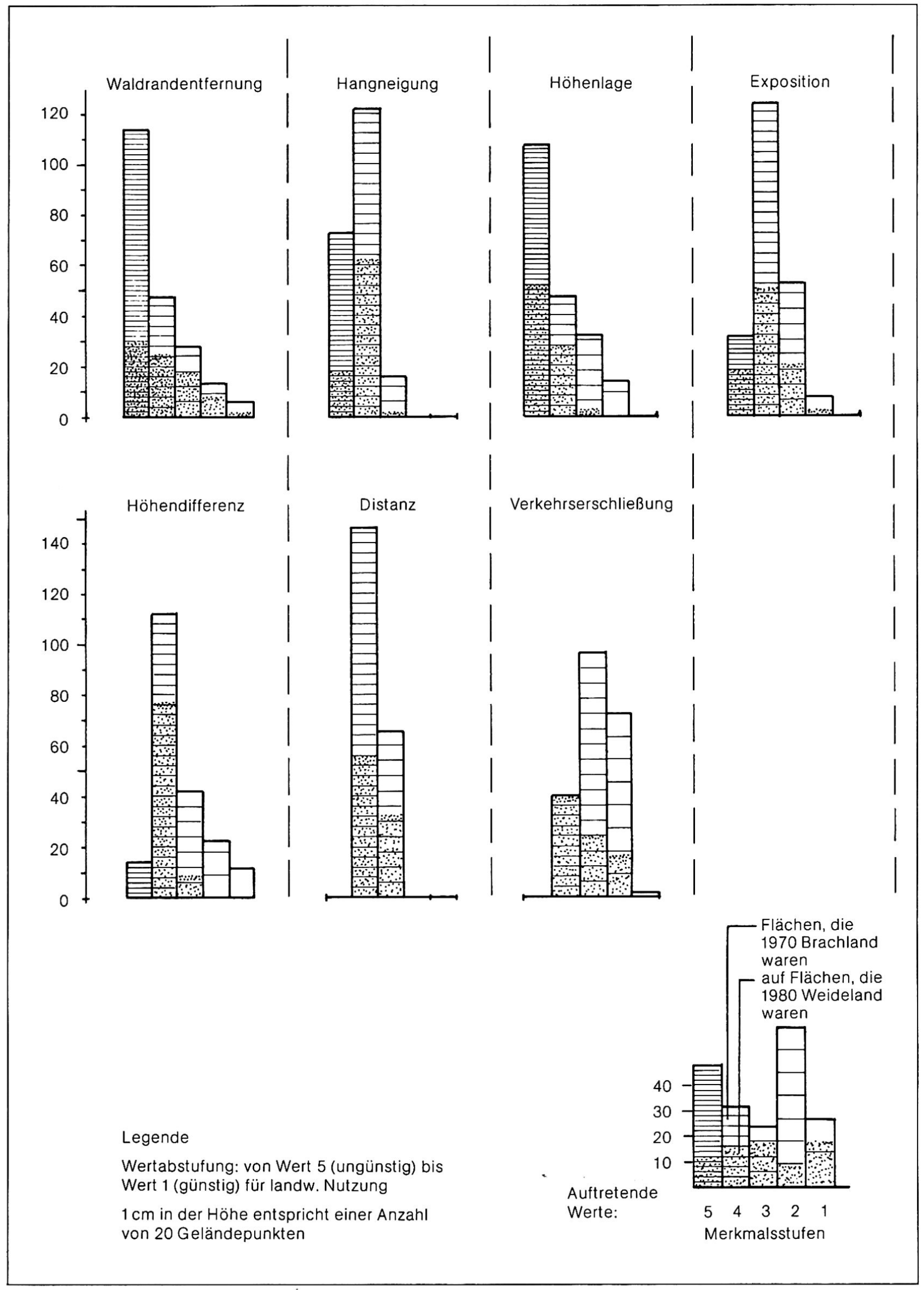

Fig. 4 Häufigkeiten der verschiedenen Merkmalsstufen (Gemeinde Niederwald/Flächen südlich der Rhone) 
Pro Merkmal wurden einheitlich fünf Merkmalsstufen unterschieden und die einzelnen Werte rasterweise erhoben (Kantenlänge $24 \mathrm{~m} \times 30 \mathrm{~m}$, bedingt durch die EDV-Ausgabegeräte). Als weiteres Merkmal wurde die Landnutzung 1970 (insbesondere Brachland/Nicht-Brachland) erfaßt. In einem nächsten Schritt wurden alle brachliegenden Rastereinheiten herausgesucht und die Werte der verschiedenen Merkmale in einer Tabelle ausgedruckt. Die Auswertung bezüglich der Häufigkeiten des Auftretens der einzelnen Merkmalsstufen gab Aufschluß über die unterschiedliche Bedeutung der einzelnen Merkmale. Es wurde mit folgenden Gewichtungen gearbeitet:

- Waldrandentfernung

Faktor 6

- Hangneigung

- Höhenlage

- Distanz zum Dorf

Faktor 5

Faktor 4

Faktor 3

- Höhendifferenz

Faktor 2

- Exposition

Faktor 1

Die Verkehrserschließung wies im Untersuchungsgebiet eine zu geringe Korrelation mit Brachland auf und wurde deshalb nicht berücksichtigt. Die gewichteten Merkmale wurden additiv verknüpft. Die Einheiten mit den höchsten Summen wurden als sehr bracheanfällig klassiert. Insgesamt wurden fünf Anfälligkeitsklassen gebildet. Die Brachlandverteilung von 1970 konnte zu 74\% mit diesen sechs naturräumlichen Merkmalen erklärt werden.

\subsection{Prognostizierung der Brachlegung und Vergleich mit Kartierung 1980}

Weil zwischen der Klasse 5 und dem effektiv brachliegenden Land eine relativ hohe Übereinstimmung bestand, konnte vermutet werden, daß die nächsten
Gebiete, die brachgelegt würden, die bisher noch nicht brachliegenden Einheiten der Klasse 5 und allmählich die Einheiten der Klasse 4 umfassen werden. Zu welchem Zeitpunkt die Flächen der Klasse 4 oder 5 aber effektiv brachfallen, kann durch das Modell nicht vorausgesagt werden.

Die Kartierung von 1980 erlaubt, die Aussagen des Modells zu überprüfen. Fig. 3 zeigt auf Rasterbasis die Zugehörigkeiten zu den Bracheanfälligkeitsklassen 4 und 5 und die 1980 aufgenommene Landnutzung. In Tab. 2 sind die Werte zusammengestellt.

Offensichtlich ist die Brachlegung nördlich und südlich der Rhone nicht gleich rasch vorangeschritten. Der Anteil an noch nicht brachgelegten Flächen der Klassen 4 und 5 ist nördlich der Rhone merklich größer (29 Einheiten) als im Südabschnitt (12 Einheiten). Trotz dieser Unterschiede in der Ausbreitungsgeschwindigkeit ist $\mathrm{zu}$ erkennen, daß tatsächlich die Einheiten in Klasse $51980 \mathrm{zu}$ einem großen Teil von der Brachlegung erfaßt wurden und bei der Klasse 4 rund die Hälfte.

Neu gegenüber dem Zustand 1970 sind die größeren Flächen an Weideland (Kap.3.3). Insgesamt werden ca. 4,4 ha ehemaliges Brachland in Niederwald wieder genutzt. Die Begehung im Gelände zeigt jedoch, daß es sich um eine sehr extensive Art von Beweidung handelt und die längerfristige Nutzung keineswegs als gesichert betrachtet werden darf. Beispielsweise wurden 1981 rund 2,2 ha dieser Fläche nicht mehr beweidet.

Das Modell 1970 gilt für die Wirtschaftsform der Mähgraswirtschaft. Das Auftreten von Weiden wirft die Frage auf, ob sich das Modell auch auf diese Bewirtschaftungsform erweitern und differenzieren

Tabelle 2 Prognostizierte und reale Brachlandausdehnung (Gemeinde Niederwald)

\begin{tabular}{|c|c|c|c|}
\hline \multicolumn{2}{|c|}{$\begin{array}{l}\text { Im Jahre } 1970 \text { für } 1980 \\
\text { prognostiziertes Brachland }\end{array}$} & \multicolumn{2}{|l|}{ realer Zustand 1980} \\
\hline & & $\begin{array}{l}\text { effektiv brach oder sehr extensiv } \\
\text { genutztes Weideland }\end{array}$ & $\begin{array}{l}\text { nicht brachliegende } \\
\text { Einheiten }\end{array}$ \\
\hline & $\begin{array}{l}\text { Einheiten als sehr brache- } \\
\text { anfällig beurteilt } \\
\text { (Klasse 5) }\end{array}$ & 267 Einheiten & 41 Einheiten \\
\hline 275 & $\begin{array}{l}\text { Einheiten als brache- } \\
\text { anfällig beurteilt } \\
\text { (Klasse } 4 \text { ) }\end{array}$ & 149 Einheiten & 126 Einheiten \\
\hline 247 & $\begin{array}{l}\text { Einheiten wenig brache- } \\
\text { anfällig } \\
\text { (Klassen } 1,2,3)\end{array}$ & 60 Einheiten & 383 Einheiten \\
\hline
\end{tabular}

1 Einheit $=720 \mathrm{~m}^{2}$ 
Tabelle 3 Entwicklung der Landnutzung 1970-1980 (Gemeinde Niederwald)

\begin{tabular}{|lllllrr|}
\hline $\begin{array}{l}\text { Landnutzung } \\
1970\end{array}$ & $\begin{array}{l}\text { Landnutzung } \\
1980\end{array}$ & $\begin{array}{l}\text { Flächen nördl. } \\
\text { der Rhone } \\
\text { absolut (ha) }\end{array}$ & \multicolumn{1}{c}{$\%$} & $\begin{array}{l}\text { Flächen südl. } \\
\text { der Rhone } \\
\text { absolut (ha) }\end{array}$ & $\%$ & \multicolumn{2}{l|}{$\begin{array}{l}\text { Flächen } \\
\text { total } \\
\text { absolut (ha) }\end{array}$} & \% \\
\hline brach $\longrightarrow$ brach & 4,2 & 9,9 & 9,7 & 31,3 & 13,9 & 18,9 \\
brach $\longrightarrow$ Mähwiesen & 2,7 & 6,3 & 0,5 & 1,6 & 3,2 & 4,4 \\
brach $\longrightarrow$ Weide & 0,0 & 0,0 & 4,2 & 13,6 & 4,2 & 13,6 \\
Mähwiesen $\longrightarrow$ Mähwiesen & 30,3 & 70,7 & 6,5 & 21,1 & 36,8 & 49,9 \\
Mähwiesen $\longrightarrow$ Weide & 0,0 & 0,0 & 2,3 & 7,4 & 2,3 & 7,4 \\
Mähwiesen $\longrightarrow$ brach & 5,5 & 12,9 & 7,7 & 24,8 & 13,2 & 17,9 \\
\hline
\end{tabular}

ließe in «voraussichtliches Mähgrasland - voraussichtliches Weideland - voraussichtliches Brachland». Fig. 4 zeigt die Histogramme der Häufigkeiten der einzelnen Merkmalsstufen des 1970 brachliegenden Landes, überlagert mit den Häufigkeiten des Weidelandes 1980. Die Berechnung ergibt bei allen Merkmalen hohe Korrelationen (Korrelationskoeffizienten zwischen 0,85 und 0,99 ). Somit ist es nicht möglich, mit dem 1970 verwendeten Satz von Merkmalen zwischen voraussichtlichem Brachland und voraussichtlichem Weideland zu differenzieren. Der Satz der Merkmale müßte durch den Einbezug sozio-ökonomischer und betriebswirtschaftlicher Merkmale ergänzt werden.

Fig. 3 gibt die Landnutzung 1980 wieder. Um ein Bild vom Ausmaß der Übereinstimmung von Prognose und realer Entwicklung zu bekommen, wurden die Flächen, die nach Modell zu den Brachlandanfälligkeitsklassen 4 und 5 gehören, ebenfalls dargestellt.

\subsection{Dynamik der Landschaftsentwicklung}

Die Bearbeitung mit Hilfe eines Rasters ermöglicht eine räumlich fein differenzierte Beurteilung von Entwicklungen. Dabei fällt die Verschiedenartigkeit der Veränderungen auf. Eine entsprechende Auswertung ist in Tab. 3 wiedergegeben. In Fig. 5 wird die Entwicklung in Form eines Flußdiagrammes zusammengefaßt.

\subsection{Folgerungen}

Eine kritische Würdigung des Modellansatzes 1970 erlaubt folgende Schlüsse:

- Die Prognostizierung des Überganges von Mähwiesen zu Brachland erfolgte weitgehend richtig.

- Eine differenzierte Behandlung der Flächen des Nord- und des Südhanges hätte die unterschiedlichen Ausdehnungsgeschwindigkeiten besser aufgezeigt.

- Das Modell ist ausgerichtet auf die Wirtschaftsform der Mähgraswirtschaft. Es läßt sich jedoch in der vorliegenden Form nicht für die Prognose bezüglich künftigen Weidelandes einsetzen (Kap. 3.3).

\section{Beurteilung und Maßnahmenkatalog}

Bei der Beurteilung der beschriebenen Prozesse stellt sich die Frage, ob diese Entwicklungen den Zielvorstellungen des Regionalen Entwicklungskonzeptes (1974/1976) bezüglich Landwirtschafts- und Landschaftsplanung entsprechen. Im Produktionskonzept heißt es, daß man sich nach längerer Diskussion im Obergoms für die Förderung einer Intensivnutzung ausgesprochen habe. Die intensive Nutzung der mechanisierbaren Flächen sollte durch eine extensive Weidenutzung der steileren Hanglagen ergänzt werden. Die beobachtete Zunahme der Beweidung im unteren Talabschnitt (Kap. 3.3) entspricht also diesem Flächenkonzept und ist somit unterstützenswert. Bereits 1974 wurde die Vergandung des Kulturlandes als "schwerwiegendster Schaden»' in der Landschaft erkannt. «Durch das Verwildern der ehemals landwirtschaftlich genutzten Flächen geht dem Tal wesentliche Substanz verloren. Der Frage, wie dieser Entwicklung entgegengesteuert werden kann, ist die Hauptaufmerksamkeit im Rahmen des Landschaftsschutzes (Landschaftspflege) zu widmen" (Reg. Entw. Konzept 1974, S. 6). Der Verlust dieser «landschaftlichen Substanz» kann sich eventuell nachteilig auf die touristische Attraktivität des Obergoms auswirken, wird doch in den Prospekten häufig mit der ursprünglichen, unverdorbenen Agrarlandschaft des Obergoms geworben. Somit sind die Folgen der beschriebenen Prozesse im Landschaftsbild quasi Maßstab für die folgende Beurteilung.

Die ökologischen Folgen der Brachlegung von Magerwiesen im Obergoms hat Bischof (1981) untersucht. Er stellt fest, daß die Vegetation, insbesondere die Blumenvielfalt in den Wiesen, durch die Brachlegung stark verarmt. Mit dem Auftreten der ersten Holzgewächse werden die ersten bedeutenderen ökologischen Auswirkungen sichtbar. Da der Boden im Obergoms sehr flachgründig ist, wird er sehr intensiv mit Wurzeln durchsetzt, so daß er in Zukunft selbst durch Stockrodungen nicht mehr urbar gemacht werden kann. Nach Bischof (1981) können die Sukzessionslinien an verschiedenen Standorten unterschiedlich verlaufen. An Nordhängen wachsen in der 
Regel Lärchen, Fichten oder Grünerlen, an Südhängen zudem Birken. Eine besondere Sukzessionsphase bildet der Zwergstrauchaufwuchs (Calluna vulgaris, Vaccinium myrtillus), der nach längerer Zeit meist in Lärchenwald übergeht. Dabei sind die Lärchenwälder sowohl ökologisch als auch für den Tourismus wertvoller zu beurteilen als der dichte Aufwuchs von Grünerlen. Die Folgen des Brachlegungsprozesses sind vor allem an den steilen Hängen in Dorfnähe ernstzunehmen. Es werden unter anderem ökologische Schäden wie die Erhöhung der Lawinen-, Erosions- und Brandgefahr vermutet, was auch die Siedlungen gefährden könnte. Die Zunahme des Brachlandes auf diesen parzellierten Flächen ist also nicht wünschenswert, wird aber bei gleichbleibenden Rahmenbedingungen weiter fortschreiten.

Schwieriger gestaltet sich die ökologische Beurteilung der Weideflächen (zweiter räumlicher Prozeß). Vor allem die Schädigung von Boden und Grasnarbe durch die Schafbeweidung wird verschiedentlich diskutiert (FEHSE 1974).

Immerhin kann die zunehmende Beweidung von ehemaligem Brachland als Landschaftspflege ${ }^{2}$ bezeichnet werden. Daß durch das neue Element der eingezäunten Weiden etwas von der «Romantik der guten alten Zeit» (Vereinig. der Verkehrsvereine 1978) verlorengeht, ist unvermeidbar. Im Frühjahr 1981 ließ sich im Obergoms eine Reihe von großen Erdrutschen beobachten. Vermutlich sind sie auf zerfallene Bewässerungskanäle, die in den Weiden natürlich nicht mehr gepflegt werden, zurückzuführen. Die Entwicklung solcher Schadenfälle sollte auf jeden Fall verfolgt werden, und es ist abzuklären, inwieweit die Beweidung eine Verbuschung des Landschaftsbildes verhindern kann. Wenn die Weiden nicht regelmäßig gesäubert werden, kann man bald das Aufwachsen von Holzgewächsen feststellen.

Der abschließende Maßnahmenkatalog ist vom Ziel bestimmt, dem Obergoms auch in Zukunft eine gesunde wirtschaftliche Basis zu erhalten. Diese Basis liegt einerseits in der Bewahrung des wertvollen Kulturlandes vor der Verwaldung und andererseits in der Erhaltung eines gepflegten Landschaftsbildes für den Tourismus, wobei einer angepaßten landwirtschaftlichen Nutzung Priorität einzuräumen ist. Aus der Erkenntnis, daß es sich im Obergoms vor allem um Strukturbrache (SuRber 1973) handelt, folgt die alte Forderung, daß Besitz- und Pachtlandarrondierungen verstärkt vorangetrieben werden müssen. Dadurch können die Rahmenbedingungen für die Beweidung der Talhänge entscheidend verbessert werden. In besonderen Fällen ist das Gesetz der Bewirtschaftungspflicht durchzusetzen, da einzelne Bauern diese Entwicklung zur extensiven Landnutzung sonst erfolgreich verhindern könnten. Auf kantonaler Ebene ist eventuell an die Ausarbeitung eines Gesetzes für zwangsweise Umlegungen zu denken. Zur Durchsetzung dieser Forderungen müssen zuerst die Planunterlagen verbessert werden.

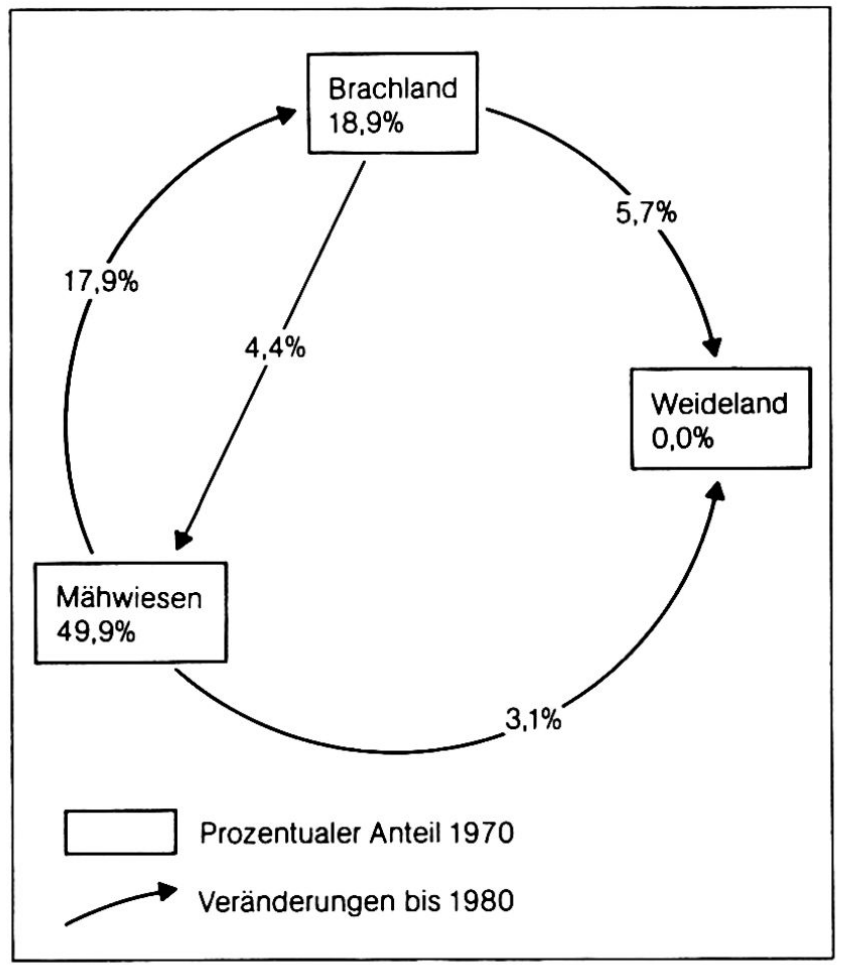

Fig. 5 Flußdiagramm der Landnutzungsentwicklung (Gemeinde Niederwald) (vgl. Tab. 3, rechte Kolonne)

Problematisch ist die Förderung der Nebenerwerbslandwirtschaft im Rahmen der Regionalen Entwicklungskonzeption als Lösung der anstehenden Probleme. LANDTwING (1979) zeigt, daß die Arbeiterbauern in den Betrieben meist Hilfsarbeiterstellen ohne Aufstiegschancen besetzen. Es ist fraglich, ob die Nachkommen, wenn die Beziehung zur Landwirtschaft einmal gelockert ist, weiter diesem Doppelerwerb nachgehen werden. Für den Tourismus sollten entlang dem Gommer Höhenweg einige Magerwiesen als Biotope geschützt und durch ein- bis zweijährigen Schnitt sichergestellt werden. Wichtiger erscheint aber im Goms die Erhaltung der charakteristischen Dorfbilder. Diese sind aus den gleichen Gründen wie das reich parzellierte Wies- und Ackerland von der Nutzungsauflassung betroffen. Die kleinen Wirtschaftsgebäude sind nur schwer rationalisierbar und stehen deshalb ungenutzt.

Bei der Durchsetzung dieser Forderungen muß beachtet werden, daß staatliche Maßnahmen allein nur selten fruchten. Ein gutes Beispiel ist die Auszahlung von Flächenbeiträgen an die Landwirtschaft, die von den Bauern zum Teil skeptisch beurteilt wird. Wie eine interessante Studie im Goms zeigt (Institut Sek 1980), sollte die ansässige Bevölkerung mit ihren sozialen Strukturen und ihren Wertvorstellungen vermehrt in die Planung einbezogen werden. 


\section{Zusammenfassung}

Im vorliegenden Bericht wird erstmals die Dynamik der Brachlandentwicklung in einer Bergregion untersucht. Dabei lassen sich zwischen 1970 und 1980 zwei räumliche Prozesse beobachten:

1. Zunahme des Brachlandes auf den ehemaligen Ackerbauflächen

2. Abnahme des Brachlandes infolge zunehmender Weidenutzung

Diese Prozesse sind ursächlich miteinander verknüpft. Am Beispiel von Niederwald werden zudem die Veränderungen mit der im Jahre 1970 mit Hilfe eines
Modelles errechneten Brachlandentwicklung verglichen. Der gewählte Modellansatz erweist sich in der Prognostizierung des Überganges von Mähwiesen zu Brachland als brauchbar. Die abschließende Beurteilung und der Maßnahmenkatalog orientieren sich an den Zielvorstellungen der Regionalplanung und an den Folgen der beiden Prozesse im Landschaftshaushalt.

\footnotetext{
' Dieser Begriff ist rein normativ und wurde im Zusammenhang mit der Brachlegung erstmals von NoHL (in KTBL 1976) untersucht.

${ }^{2}$ Landschaftspflege muß hier im Sinne einer Offenhaltung der Flur verstanden werden.
}

\section{Literaturverzeichnis}

Bischof, N.: Pflanzensoziologische Untersuchungen von Sukzessionen aus gemähten Magerrasen in der subalpinen Stufe der Zentralalpen. Diss. Basel 1981.

Dietl, W.: Die Kartierung des Pflanzenstandortes und die futterbauliche Nutzungseignung von Naturwiesen. Zürich 1979.

FeHSE, R.: Gedanken zum Einsatz des Schafes und der Ziege in der Landschaftspflege im Berggebiet. In: Schw. Landw. Monatshefte, 1974, S. 337-349.

Gerber, M., Gianquet, B.: Binn - Landnutzung im Tal. Manusk. Geogr. Inst. Univ., Bern 1978.

Gresch, P.: Wandel in Wirtschaftsform und Landschaftsbild im inneralpinen Lebensraum am Beispiel des oberen Goms. Dipl. Arb. Geogr. Inst. Univ., Zürich 1971.

Gresch, P.: Vergandete Agrarareale im Oberen Goms. In: Geogr. Helv., 1972, S. 145-147.

Hottes, K.H.: Geographie. In: Brockhaus-Enzyklopädie, Bd. 22. Wiesbaden 1975.

Institut F. Sozialethik Des SeK: Entwicklung im Stadtquartier und in der Bergregion. Bern 1979.

JULEN, S.: Dauerbrachland in einem touristisch stark frequentierten Gebiet. Dipl. Arb. Geogr. Inst. Univ., Zürich 1979.

KLOPSCH, U.: Anthropogeographische Veränderungen und deren Ursachen in den Obergommer Gemeinden Münster, Reckingen, Gluringen, Ritzingen, Biel und Selkingen seit der Jahrhundertwende. Diss. Kiel 1969.
KTBL: Brachflächen in der Landschaft. Münster-Hiltrup 1976.

Landtwing, K.: Die Arbeiterbauern des Lonza-Werkes Visp und ihre Kulturlandschaft. Diss. Zürich 1979.

Lichtenberger, E.: Die Sukzession von der Agrarzur Freizeitgesellschaft in den Hochgebirgen Europas. In: Festschrift A. Leidlmayr, Innsbruck 1979.

MAtter, M.: Wertsystem und Innovationsverhalten. Diss. Zürich 1978.

Monheim, F.: Agrargeographie der westlichen Alpen. Gotha 1954.

Moos, F., Herot, S.: Möglichkeiten zur Verhinderung oder Beseitigung der Brachlegung von Landw. Grenzertragsstandorten. Ebikon 1979.

Regionales Entwicklungskonzept Goms, Brig $1974 / 76$.

Roten, E.: Die Landwirtschaft im Oberwallis. In: Die Grüne, 1969, S. 1-17.

Surber, E. ET AL.: Das Brachlandproblem in der Schweiz. Berichte EAFV, Birmensdorf 1973.

Surber, E. ET AL.: Die Kartierung von Brachland. Berichte EAFV, Birmensdorf 1979.

Vereinigung der Verkehrsvereine Goms: Touristenprospekt Goms. Münster 1981. 